\title{
Melanoma-Associated Spongiform Scleropathy Adjacent to a Choroidal Nevus
}

\author{
Alexander Schneider ${ }^{a}$ Peter R. Pavan ${ }^{a}$ Curtis E. Margo ${ }^{a, b} \quad$ Jasmina Bajric ${ }^{a}$ \\ ${ }^{a}$ Morsani College of Medicine, Department of Ophthalmology, University of South Florida, Tampa, FL, USA; \\ ${ }^{b}$ Morsani College of Medicine, Department of Pathology and Cell Biology, University of South Florida, Tampa, FL, USA
}

\section{Established Facts}

- Melanoma-associated spongiform scleropathy is a degenerative change of sclera thought to be exclusively linked to a contiguous choroidal or ciliary body melanoma.

- There is one reported case of melanoma-associated spongiform scleropathy associated with oculodermal melanocytosis and a non-contiguous primary orbital melanoma.

\section{Novel Insights}

- Melanoma-associated spongiform scleropathy was noted contiguous to a benign choroidal nevus in an eye harboring a distinct and separate choroidal-ciliary body melanoma (and without associated scleral degeneration).

- Melanoma-associated spongiform scleropathy is not exclusively related to posterior uveal melanoma.

\section{Keywords}

Choroidal nevus · Melanoma-associated spongiform scleropathy $\cdot$ Nevus $\cdot$ Scleral degeneration

\begin{abstract}
Melanoma-associated spongiform scleropathy contiguous to a choroidal nevus was an incidental finding in a 57-yearold woman whose eye was removed for a separate choroidal-ciliary body melanoma. All previously reported cases of melanoma-associated spongiform scleropathy, except for one, have been found adjacent to posterior uveal melanoma. The mechanism of scleral degeneration in melanomaassociated spongiform scleropathy is unknown. Few cases of
\end{abstract}

\section{KARGER}

(c) 2018 S. Karger AG, Basel

E-Mail karger@karger.com

www.karger.com/oop posterior uveal nevi have been reported since the description of spongiform scleropathy, making assessment of the specificity of the degeneration to melanoma alone difficult. The presence of melanoma-associated scleropathy adjacent to a choroidal nevus indicates that the condition is not exclusively linked to posterior uveal melanoma.

(c) 2018 S. Karger AG, Basel

\section{Introduction}

A peculiar degenerative change of scleral collagen associated with contiguous melanoma of choroid and/or ciliary body was initially reported by Stefani at the 1989 
Fig. 1. A $6 \times 4.5 \mathrm{~mm}$ oval hypopigmented lesion is present temporal to the right macula. A large choroidal-ciliary body melanoma creates the crescentic shadow nasally.

Fig. 2. A large dome-shaped melanoma of the choroid and ciliary body is present nasally (hematoxylin-eosin; scale bar, 2.7 $\mathrm{mm}$ ). Inset The sclera contiguous to the melanoma is normal (hematoxylin-eosin; scale bar, $350 \mu \mathrm{m})$.
1

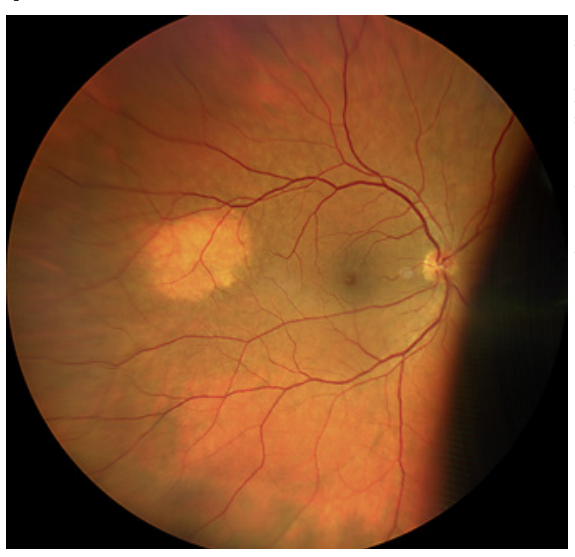

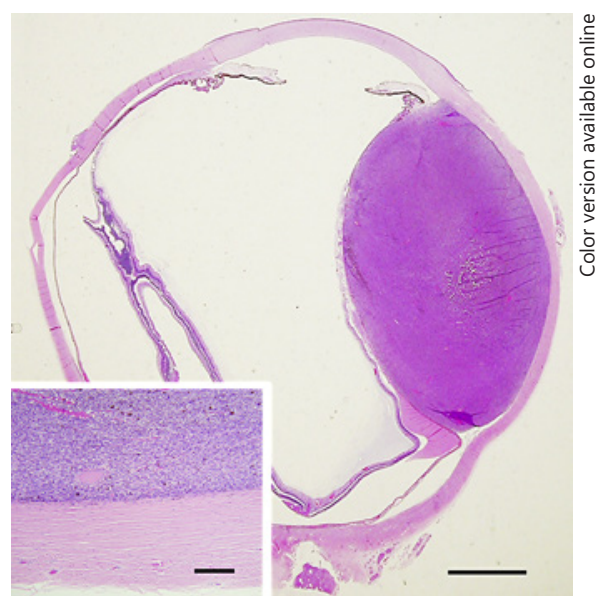

European Ophthalmic Society meeting [1]. The condition was called melanoma-associated spongiform scleropathy and has been subsequently found in $11-33 \%$ of eyes harboring choroidal and ciliary body melanomas [1-3]. The condition is thought specific to choroidal-ciliary body melanoma and has not been described in benign tumors or metastatic cancers to the eye. A single case of melanoma-associated spongiform scleropathy has been reported in oculodermal melanocytosis in an eye removed for primary orbital melanoma [4]. Although this scleral aberration is relatively common in posterior uveal melanoma - and not microscopically subtle - it was not considered a nosologic entity until the end of the 20th century. Since then, studies have examined what pathologic mechanisms might be involved in the process, and if they could impact prognosis $[5,6]$. We report melanoma-associated spongiform scleropathy contiguous to a choroidal nevus, in an eye removed for an anatomically separate mixed cell type melanoma of choroid and ciliary body.

\section{Case Report}

A 57-year-old woman was referred for management of a large choroidal-ciliary body tumor of the right eye. She had noted new floaters in her vision for the past several months with progressive blurry vision, but she sought attention when she noticed a brown spot on the nasal sclera in the right eye. Her past ocular and medical history were noncontributory. She had not had an ocular examination for years, and there were no previously documented ocular abnormalities. Her vision in the affected right eye was 20/100. Relevant findings on exam included a large $16 \times 8 \mathrm{~mm}$ brown dome-shaped mass present nasally in the peripheral choroid and ciliary body. An oval $6 \times 4.5 \mathrm{~mm}$ hypopigmented lesion was noted temporal to the macula (Fig. 1). Its thickness could not be reliably measured by ultrasound. A 1-2 mm flat brown episcleral lesion (external to the large choroid-ciliary body tumor) was present $1 \mathrm{~mm}$ anterior to the medial rectus. Otherwise there were no other relevant findings in either eye. The patient opted for enucleation, which was performed without complication.

\section{Results}

Applicable anatomic findings included a mixed cell type choroidal-ciliary body melanoma (Fig. 2). Tumor extended to the episcleral surface through an emissary canal, but sclera contiguous to melanoma was normal (Fig. 2, inset). The hypopigmented lesion temporal to the macula consisted of bland spindle-shaped melanocytes without mitotic activity. The tumor did not exceed 250 $\mu \mathrm{m}$ in thickness. The inner sclera adjacent to the lesion displayed splayed and fragmented collagen, reminiscent of shredded wheat (Fig. 3). This region stained slightly less intensely with Alcian blue, colloidal iron, and periodic acid-Schiff compared to outer sclera and sclera adjacent to the large choroidal-ciliary body melanoma (Fig. 4).

\section{Discussion}

Thousands of eyes harboring posterior segment uveal melanomas had been examined histologically for decades without recognizing that alterations in contiguous scleral collagen was something other than artifact. The abnormality is relatively common and not microscopically subtle. Although the pathogenesis of melanoma-associated spongiform scleropathy is not well understood, it is considered a condition found exclusively with posterior uve- 


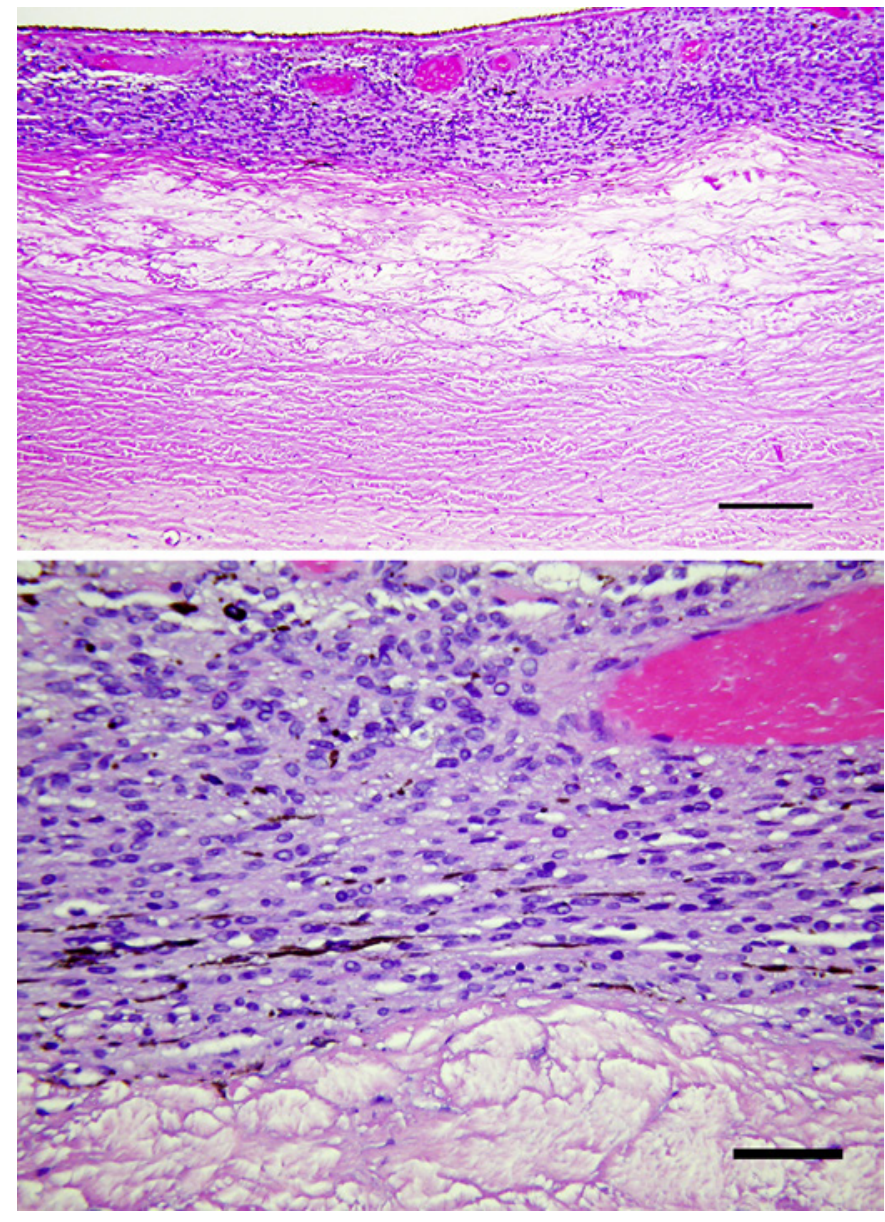

Fig. 3. The upper panel shows the macular nevus composed of spindle-shaped nuclei with bland vesicular chromatin. The sclera contiguous to the nevus appears pale relative to the outer sclera (hematoxylin-eosin; scale bar, $100 \mu \mathrm{m}$ ). The lower panel shows the nevus in higher magnification. The collagen fibers of the inner sclera are fragmented and splayed apart (hematoxylin-eosin; scale bar, $300 \mu \mathrm{m})$.

al melanoma, as its name explicitly indicates. One study of 218 eyes with choroidal and ciliary body melanoma found 73 tumors with melanoma-associated spongiform scleropathy (33\%) [1]. That study also included 45 normal and tumor-bearing eyes which did not display the scleropathy. None of the eyes, however, included a choroidal nevus. Subsequently, Ranjit and associates [4] reported widespread spongiform scleropathy in a patient with oculodermal melanocytosis after the eye was removed for a primary orbital melanoma. In that case report, affected sclera was contiguous with choroidal melanocytosis and anatomically remote from the orbital melanoma.
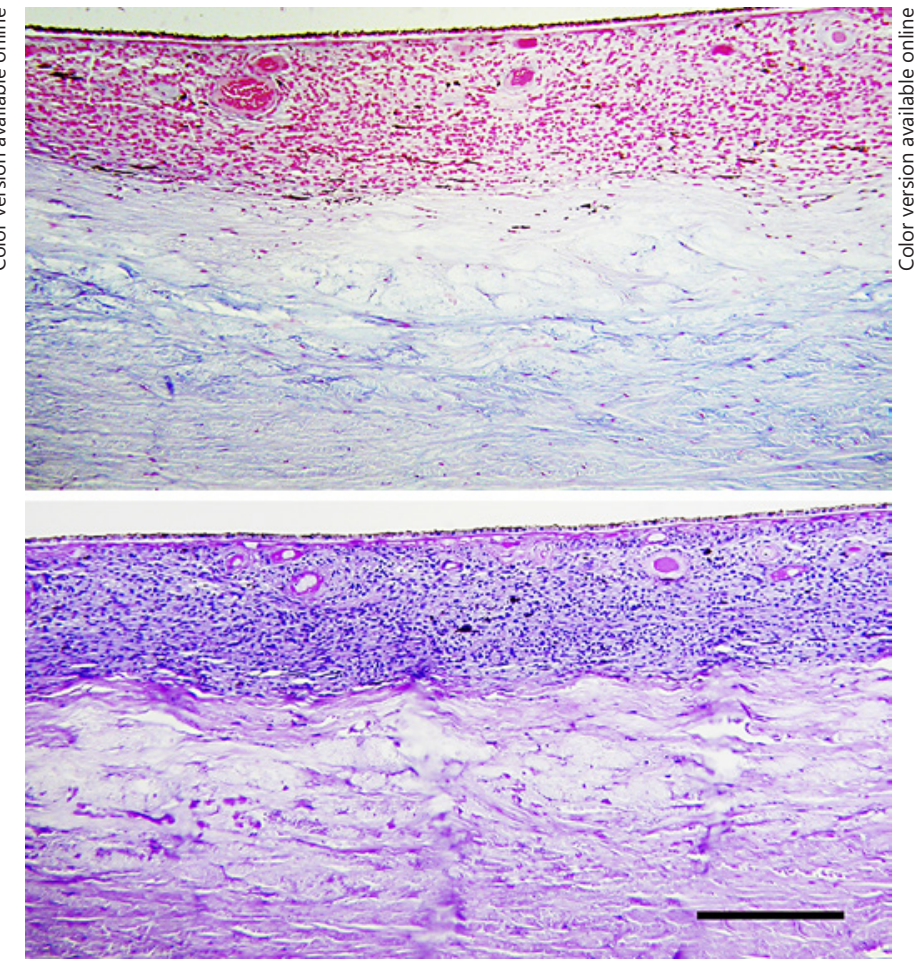

Fig. 4. Alcian blue stain above and periodic acid-Schiff (PAS) below shows diminished stain intensity in the sclera adjacent to the choroidal nevus (scale bar, $180 \mu \mathrm{m}$ ).

Choroidal nevi are common with an estimated prevalence among white adults in the United States of 4.6-7.9\% [7]. Despite nearly 9 million choroidal nevi on average being present in persons in the United States at any time, there are few studies dealing with their histopathology [8]. Most choroidal nevi are discovered incidentally on eye examination and monitored clinically. It may be premature to consider spongiform scleropathy as a degenerative process associated exclusively with posterior uveal melanoma. A study involving a substantial number of choroidal nevi that addresses the light microscopic appearance of adjacent sclera is needed to shed light on the specificity of spongiform scleropathy.

There is an apparent paradoxical observation that specific and total glycoasaminoglycans are found in higher concentrations within melanoma-associated scleropathy than normal sclera through biochemical assay [5], yet when histochemical stains for mucosubstances and polysaccharides are applied, there is reduced stainability [1]. Our findings are consistent with reports of reduced reactivity of Alcian blue, PAS, and colloidal iron in the area of scleral degeneration (Fig. 4) [1]. Further insights into the 
physical-chemical properties of this conditions are needed to clarify this apparent contradiction of findings.

In summary, the small melanocytic nevus found adjacent to melanoma-associated scleropathy reported herein was thoroughly sampled (exhaustion of the lesion in the paraffin block) and showed no evidence of malignant transformation. Why this association exists is speculative. If the degenerative process is due to prolonged contact of melanocytes with adjacent sclera, which we suspect might be the case, there is yet little corroborating evidence to support this hypothesis other than presence in a single case of oculodermal melanocytosis [4].

\section{Statement of Ethics}

The authors have no ethical conflicts to disclose.

\section{Disclosure Statement}

The authors have no conflicts of interest to declare.

\section{Author Contributions}

Dr. Alex Schneider and Dr. Curtis Margo were responsible for the creation of the manuscript. Dr. Margo was the attending pathologist who reviewed the case. Dr. Peter Pavan and Dr. Jasmina Bajric both cared for the patient and edited the manuscript.

\section{References}

1 Alyahya GA, Heegaard S, Prause JU. Characterization of melanoma associated spongiform scleropathy. Acta Ophthalmol Scand. 2002 Jun;80(3):322-6.

2 Alyahya GA. Melanoma associated spongiform scleropathy: characterization, biochemical and immunohistochemical studies. Acta Ophthalmol. 2008;Oct;86 Thesis 3:1-21.

3 Robert F. Chapter 4: The Sclera. In: Heegaard S, Grosssnikluas HE, editors. Eye Pathology. Heidelberg: Springer; 2015. pp. 155-72.
4 Ranjit RU, Leyngold IM, Margo CE. Melanoma-associated spongiform scleropathy in oculodermal melanocytosis with primary orbital melanoma. Ocul Oncol Pathol. 2016 Oct; 2(4):276-9.

5 Alyahya GA, Ribel-Madsen SM, Heegaard S, Prause JU, Trier K. Melanoma-associated spongiform scleropathy: biochemical changes and possible relation to tumour extension. Acta Ophthalmol Scand. 2003 Dec;81(6): 625-9.
6 Alyahya GA, Kolko M, Prause JU, Nielsen BS, Wang J, Heegaard S. Matrix metalloproteinase-2 is expressed in melanoma-associated spongiform scleropathy. Invest Ophthalmol Vis Sci. 2008 Jul;49(7):2806-11.

7 Singh AD, Kalyani P, Topham A. Estimating the risk of malignant transformation of a choroidal nevus. Ophthalmology. 2005 Oct: 112(10):1784-9.

8 Chien JL, Sioufi K, Surakiatchanukul T, Shields JA, Shields CL. Choroidal nevus: a review of prevalence, features, genetics, risks, and outcomes. Curr Opin Ophthalmol. 2017 May;28(3):228-37. 